\section{Características das crianças menores de cinco anos atendidas em serviços de atenção básica em dois municípios do nordeste brasileiro}

\section{The characteristics of children under five years of age receiving basic care services in two municipalities in the Northeast region of Brazil}

Juvenal Soares Dias da Costa 1

Juraci Almeida Cesar 2

Ana Paula Weber 3

Anderson da Silva Garcez 4

Carlise Rigon Dalla Nora 5

Helena Beatriz Rower 6

Vanessa Kolling 7

1,3-7 Programa de Pós-Graduação em Saúde Coletiva. Universidade do Vale do Rio dos Sinos. Av. Unisinos, 950. São Leopoldo, RS, Brasil. CEP: 93.022-000. E-mail: episoares@terra.com.br

2 Programa de Pós-Graduação em Saúde Coletiva. Universidade Federal do Rio Grande. Rio Grande, RS, Brasil.

\begin{abstract}
Objectives: to describe the prevalence of health service consultations with a doctor or a nurse among children under five years of age in the municipalities of Caracol and Anisio de Abreu, Piaui and to identify associated factors.

Methods: a population-based cross-sectional study was carried out with children aged between 0 and 59 months. Data was collected using questionnaires between July and September 2008. Poisson's Regression and the hierarchized model were used for multivariate analysis.

Results: among the 1640 children included in the study, the prevalence of health service consultations in Caracol was 44.2\% (CI95\% 40.1-47.4) and 48.5\% (CI95\% 44.8-52.1) in Anisio de Abreu. After adjustment for any confounding factors, consultation was found to be associated with higher household income and shorter distance from the health service in Caracol. In Anisio de Abreu, the outcome was associated with water supply and the child's age.

Conclusions: the analysis found differences in health care related to socioeconomic conditions between the municipalities, indicating inequities in the health service.
\end{abstract}

Key words Health services accessibility, Equity in health, Child health

\section{Resumo}

Objetivos: descrever a prevalência de consultas nos serviços de saúde com médico ou enfermeiro em crianças menores de cinco anos de idade nos municipios de Caracol e Anísio de Abreu, Piaui e identificar os fatores associados.

Métodos: trata-se de estudo transversal de base populacional com crianças de 0 a 59 meses. A coleta de dados foi realizada mediante aplicação de questionários entre julho e setembro de 2008. Utilizou-se para análise multivariada a Regressão de Poisson e o modelo hierarquizado.

Resultados: entre as 1640 crianças incluidas no estudo, a prevalência de consultas nos serviços de saúde em Caracol foi de 44,2\% (IC95\% 40,1-47,4) e em Anisio de Abreu foi de 48,5\% (IC95\% 44,8-52,1). Após ajuste para eventuais fatores de confusão, consultar esteve associado com maior renda familiar e menor distância do serviço de saúde em Caracol. Em Anísio de Abreu, o desfecho associou-se com abastecimento de água e idade das crianças.

Conclusões: a análise encontrou diferenças nos cuidados à saúde relacionados às condições socioeconômicas entre os municípios, apontando para iniquidades no sistema de saúde.

Palavras-chave Acesso aos serviços de saúde, Equidade em saúde, Saúde da criança 


\section{Introdução}

Os marcadores de saúde infantil são fortemente influenciados pelas condições de vida. ${ }^{1,2}$ Nutrição, condições de habitação, poder aquisitivo, educação e disponibilidade aos serviços de saúde são componentes fundamentais no meio em que as crianças vivem. ${ }^{3}$

As ações de saúde destinadas às crianças consistem em medidas voltadas para a prevenção e recuperação da saúde que exigem recursos de baixa complexidade e que provocam impacto positivo nas condições de vida da população infantil. Assim, preconiza-se o acompanhamento do crescimento e desenvolvimento, incentivo à amamentação, ampliação da cobertura de agentes imunizantes, cuidados com doenças respiratórias e doenças diarréicas. Portanto, entre o elenco de atividades desenvolvidas em atenção primária a saúde os cuidados com a população infantil são prioritários.

Estudos têm mostrado que a oferta de cuidados na atenção primária em saúde pode ser determinante para melhorar as condições de saúde da população.4-6

$\mathrm{Na}$ Região Nordeste do Brasil, apesar dos evidentes avanços nos indicadores de saúde, os coeficientes de mortalidade infantil são mais elevados que no restante do país. ${ }^{7}$ Essa realidade é determinada por problemas nas condições socioeconômicas, incluindo deficiência na oferta de serviços de saúde. Desta forma, estudos sobre o acesso e a utilização de serviços de saúde podem contribuir para se verificar a disponibilidade de oferta e equidade da assistência. 8,9

Os pequenos municípios, que se localizam distante dos grandes centros urbanos e que são pobres, geralmente apresentam estrutura em saúde insuficiente e carecem de profissionais qualificados para oferecer saúde do ponto de vista coletivo. 10 Sendo assim, o presente estudo teve como objetivo verificar a prevalência de consulta nos serviços de saúde com médico ou enfermeiro nos três meses anteriores à entrevista em crianças menores de cinco anos em dois municípios pobres do Piauí.

\section{Métodos}

O presente artigo é parte de um projeto mais amplo que tinha por objetivo avaliar indicadores básicos de saúde materno-infantil nos municípios de Caracol e Anísio de Abreu, Piauí. Incluiu um estudo transversal de base populacional realizado pela Universidade Federal do Rio Grande, no período de julho a setembro de 2008. A população alvo foi constituída por todas as crianças com idades até 59 meses residentes nas áreas urbana e rural destes municípios.

Estes dois municípios estão localizados no sul do Estado do Piauí, aproximadamente $600 \mathrm{~km}$ de Teresina, a capital. Segundo estimativas do Instituto Brasileiro de Geografia e Estatística (IBGE), 11 a população de Caracol e de Anísio de Abreu era de 10.212 e 9.098 habitantes, respectivamente. O Índice de Desenvolvimento Humano (IDH) do município de Caracol era de 0,59 e o Produto Interno Bruto (PIB) per capita de R $\$ 2.618,38.12$ O município contava com cinco estabelecimentos de saúde, sendo quatro públicos e um privado. Em Anísio de Abreu, o IDH era de 0,63 e o PIB per capita de R $\$ 2.469,67$ (2008).12 O município contava com quatro estabelecimentos de saúde, todos públicos. Segundo o Sistema de Informações sobre Mortalidade, a mortalidade infantil foi muito variável em Caracol e em Anísio de Abreu. Entre 2006 e 2010, as maiores taxas de mortalidade infantil atingiram 43,8 óbitos por mil em Caracol em 2008 e 21,7 em Anísio de Abreu em 2009.13 O IDH dos municípios pesquisados figurava entre os mais baixos do país. Em 2008, quando este estudo foi realizado, o Programa Bolsa Família do governo federal atendia cerca de dois terços de todas as famílias em cada um destes municípios. Cerca da metade da população vivia abaixo da linha da pobreza. 11

Este estudo incluiu todas as crianças menores de cinco anos residentes nestes municípios no período da coleta de dados. Foram excluídas mães que apresentavam limitações cognitivas e/ou físicas que impediam a aplicação do questionário. Ao final da coleta dos dados, uma mãe que possuía duas crianças menores de cinco anos foi excluída.

Foi calculado posteriormente que o número de crianças encontradas era suficiente para garantir nível de confiança de $95 \%$, poder de $80 \%$, e exposições variando de $20 \%$ a $80 \%$, permitindo identificar uma razão de risco de 1,7.

Em ambos os municípios, as quadras nas áreas urbanas foram numeradas, e nas áreas rurais, os limites foram delimitados por estradas, riachos e montanhas. Os domicílios foram visitados e as crianças selecionadas. Foi realizado treinamento com dez entrevistadores, os quais realizaram a aplicação dos questionários. Ao final do treinamento, foi realizado um estudo piloto no município de São Raimundo Nonato, Piauí. A coleta de dados ocorreu a partir da aplicação de questionários padronizados e pré-codificados às mães ou as pessoas responsáveis pela guarda obtendo-se informações referentes à criança e a família. O controle de qualidade foi 
realizado durante a coleta, por meio de repetição parcial de 5\% das entrevistas ou supervisão direta.

O principal desfecho do estudo foi consultar nos serviços de saúde com médico ou enfermeiro nos três meses anteriores à entrevista.

As variáveis independentes foram classificadas como: geográficas (município; área urbana ou rural); características maternas e paternas (cor da pele referida pela mãe; escolaridade da mãe; escolaridade do pai; idade da mãe; número de filhos); variáveis socioeconômicas (renda familiar mensal em salários mínimos; abastecimento de água; possuir luz elétrica; distância do serviço de saúde); biológicas da criança (sexo; idade, cor da pele referida; estado nutricional); suporte/organização da família (receber Bolsa Família; possuir cartão da criança); indicadores de gravidade (hospitalização no último ano; morte de criança menor de cinco anos no ano anterior à entrevista na residência).

Para classificação do estado nutricional foram tomadas as medidas de peso e estatura ou comprimento, este se as crianças eram menores de dois anos de idade. O peso foi obtido por meio de balança portátil com precisão de 100 gramas fornecidas pelo Fundo das Nações Unidas para a Infância (UNICEF) e instaladas no próprio domicilio da criança. Para o comprimento foi utilizado infantômetro Harpenden com precisão de $1 \mathrm{~mm}$ (Holtain, Crymych, UK) e técnica padronizada, enquanto a altura foi medida usando estadiômetros de alumínio com precisão de $1 \mathrm{~mm}$. Assim, o estado nutricional das crianças foi classificado pelo índice de massa corporal (IMC) obtido a partir do indicador peso/altura por meio do programa Anthro. 14 Os valores obtidos neste cálculo foram convertidos em escore-z de acordo com as Curvas de Crescimento da Organização Mundial da Saúde (OMS). Considerou-se como portadora de excesso de peso a criança que apresentou escore-z para IMC $>+1 \mathrm{em}$ relação à mediana da população de referência. Assim, nesta categoria foram incluídas todas as crianças com risco de sobrepeso $(>+1$ a +2$)$, sobrepeso $(>+2 \mathrm{a}+3)$ e obesidade $(>+3) .15$

A entrada dos dados foi realizada através do Programa Epi Info versão 6.04, com dupla entrada, para posterior comparação dos bancos de dados e correção dos possíveis erros de digitação. Posteriormente ocorreu a limpeza do banco de dados a partir do Programa Stata versão 11.0. Durante o processamento dos dados, foram checadas consistência e validade interna dos dados pelos pesquisadores responsáveis pelo estudo.

A análise descritiva dos dados foi realizada através do programa Stata versão 11.0, comparando- se os municípios. Foi realizada análise bruta estratificada por municípios, pois embora aparentemente fossem semelhantes, apresentavam diferenças na composição sociodemográfica.

As variáveis que atingiram níveis de significância $<0,20$ foram incluídas no modelo ajustado hierarquizado ${ }^{16}$ mediante Regressão de Poisson. ${ }^{17} \mathrm{O}$ modelo de análise foi hierarquizado colocando-se no nível mais distal as variáveis geográficas. O segundo nível foi constituído pelas características maternas e paternas; pelas variáveis socioeconômicas e pelas variáveis biológicas da criança. No terceiro nível estavam as variáveis referentes à organização da família seguidas pelos indicadores de gravidade (quarto nível). Todos os níveis determinavam os níveis inferiores e tinham efeito direto sobre o desfecho (Figura 1).

O projeto de pesquisa foi submetido e aprovado pelo Comitê de Ética em Pesquisa da Faculdade de Medicina da Universidade Federal de Pelotas (CEPAS/UFPel). Todos os participantes assinaram o Termo de Consentimento Livre e Esclarecido (TCLE).

\section{Resultados}

Entre as 1640 crianças menores de cinco anos participantes do estudo, 755 (46,0\%; IC95\% 43,6 - 48,4) consultaram em serviços de saúde nos três meses anteriores à entrevista. Do total de 928 crianças do município de Caracol, 410 (44,2\%; IC95\% 40,1 $47,4)$ consultaram em serviços de saúde nos três meses anteriores à entrevista e em Anísio de Abreu observou-se que do total de 712 crianças participantes, 345 (48,5\%; IC95\% 44,8 - 52,1) tinham consultado.

A maioria dos indivíduos incluídos no estudo, em ambos os municípios, era de cor da pele parda/mulata, tinha escolaridade materna e paterna de até oito anos e as mulheres tinham de 20 a 29 anos. Mais de $80 \%$ das famílias possuíam renda inferior a dois salários mínimos, cerca de metade não tinha abastecimento de água em sua residência e a maioria recebia fornecimento de luz elétrica e residia a menos de três quilômetros de distância do serviço de saúde. Em relação às características biológicas das crianças foi constatado um predomínio de meninas em Caracol e de meninos em Anísio de Abreu. Foi confirmado um maior percentual de crianças de cor parda. Em ambos os municípios a prevalência de sobrepeso/obesidade foi superior ao de desnutrição. Entretanto, destacou-se que a prevalência de desnutrição foi de aproximadamente $17 \%$ nas duas localidades. Quanto às variáveis que 
Figura 1

Modelo hierarquizado de análise.

$1^{\circ}$ Nível

\begin{tabular}{|c|}
\hline $\begin{array}{c}\text { Variáveis geográficas } \\
\text { Município } \\
\text { Área (urbana ou rural) }\end{array}$ \\
\hline
\end{tabular}

$2^{\circ}$ Nível

Características maternas/paternas

Cor da pele da mãe

Escolaridade mãe

Escolaridade pai

Idade da mãe

Número de filhos
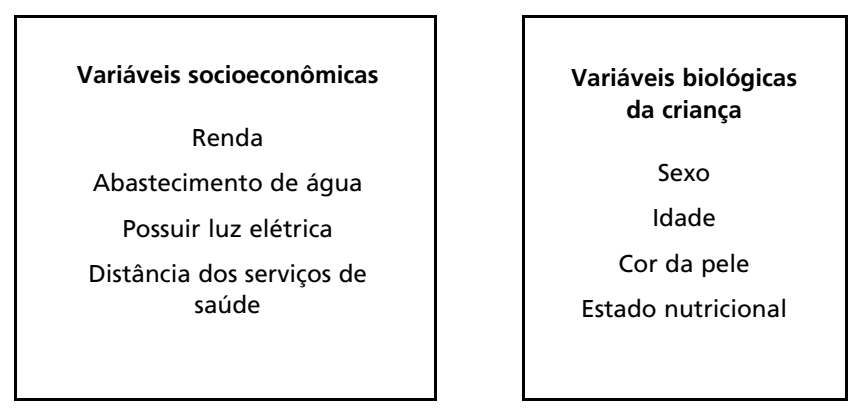

$3^{\circ}$ Nível

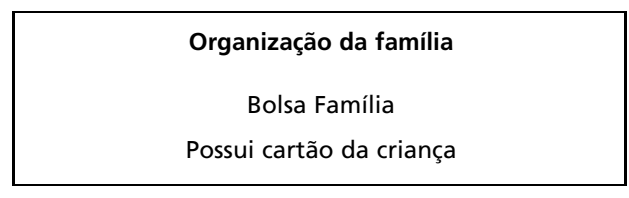

$4^{\circ}$ Nível

Indicadores de gravidade

Hospitalização no último ano

Morte de criança com menos de cinco anos

Desfecho

Consultar em serviço de saúde nos três meses anteriores à entrevista 
caracterizaram a organização da família, em ambos os municípios, verificou-se que quase metade recebia bolsa família e mais de $90 \%$ apresentaram cartão da criança. Nos dois municípios a prevalência de internações hospitalares no último ano foi superior a $10 \%$, enquanto a ocorrência de óbitos nas crianças menores de cinco anos foi inferior a $1 \%$ (Tabela 1).

Na comparação entre os municípios, constatouse que em Caracol a maior parte da população residia na área rural, referiu cor da pele como parda/mulata e preta, a escolaridade das mães foi mais baixa e o percentual de multíparas foi mais elevado. Em Caracol também foi observado maior número de famílias sem abastecimento de água e sem luz elétrica na casa. O percentual de pessoas que residiam a mais de três quilômetros foi mais elevado em Caracol (Tabela 1).

$\mathrm{Na}$ análise bruta, em Caracol, foram observadas maiores prevalências de consulta em serviços de saúde nas crianças com renda familiar mensal com três ou mais salários mínimos e que residiam a menos de três quilômetros de serviços de saúde. Estas diferenças foram confirmadas pelos intervalos de confiança e pelos testes estatísticos. Algumas variáveis foram conduzidas ao modelo, mas sem significância estatística. Foi constatado que crianças com pais de escolaridade mais elevada, que tinham fornecimento de luz elétrica na casa, que possuíam cartão da criança e que haviam sido hospitalizadas no último ano apresentaram maiores prevalência de consulta em serviços de saúde. Por sua vez, as crianças cujas mães apresentavam 30 anos ou mais, de famílias com número maior de filhos e com idade até 23 meses consultavam menos nos serviços de saúde (Tabela 2).

Em Anísio de Abreu, as maiores prevalências de consulta em serviços de saúde foram constatadas nas crianças com mães de escolaridade mais elevada, entre as mães mais jovens (13-19 anos) e em famílias que possuíam abastecimento de água do domicílio. Também entraram no modelo ajustado algumas variáveis que mostraram maiores prevalência do desfecho, como morar na zona urbana e possuir luz elétrica. As crianças que tinham três ou mais irmãos e com até 11 meses de idade consultaram menos (Tabela 2).

Em Caracol, após ajuste permaneceram no modelo as variáveis: renda familiar e distância do serviço de saúde. As variáveis idade da mãe e hospitalização no último ano apresentaram níveis de significância limítrofes. As crianças de famílias com renda mensal de três ou mais salários mínimos mensais e que moravam a menos de $3 \mathrm{~km}$ apresentaram maior prevalência de consulta em serviços de saúde nos três meses anteriores à entrevista. As crianças cujas mães tinham 30 anos ou mais consultaram menos (Tabela 3).

No município de Anísio de Abreu se verificou que as crianças cujas residências possuíam abastecimento de água no terreno consultaram mais nos serviços de saúde nos três meses anteriores à entre-

\section{Tabela 1}

Distribuição da amostra de acordo com as variáveis para consulta em serviço de saúde nos últimos três meses de acordo com variáveis geográficas, demográficas, socioeconômicas, biológicas da criança, indicadoras de gravidade e de organização da família. Caracol e Anísio de Abreu, Piauí, Brasil, 2008 (N=1640).

\begin{tabular}{|c|c|c|c|c|c|}
\hline \multirow[t]{2}{*}{ Variáveis } & \multicolumn{2}{|c|}{ Caracol } & \multicolumn{2}{|c|}{ Anísio de Abreu } & \multirow{2}{*}{$p^{a}$} \\
\hline & $\mathrm{n}$ & $\%$ & $\mathrm{n}$ & $\%$ & \\
\hline \multicolumn{6}{|l|}{ Geográficas } \\
\hline Área $(\mathrm{N}=1640)$ & & & & & $<0,001$ \\
\hline Rural & 574 & 61,9 & 347 & 48,7 & \\
\hline Urbana & 354 & 38,1 & 365 & 51,3 & \\
\hline \multicolumn{6}{|l|}{ Características maternas/paternas } \\
\hline Cor da pele da mãe $(N=1640)$ & & & & & 0,009 \\
\hline Branca & 101 & 10,9 & 114 & 16,1 & \\
\hline Parda/mulata & 794 & 85,5 & 577 & 81,0 & \\
\hline Preta & 33 & 3,6 & 21 & 2,9 & \\
\hline
\end{tabular}


Distribuição da amostra de acordo com as variáveis para consulta em serviço de saúde nos últimos três meses de acordo com variáveis geográficas, demográficas, socioeconômicas, biológicas da criança, indicadoras de gravidade e de organização da família. Caracol e Anísio de Abreu, Piauí, Brasil, 2008 (N=1640).

\begin{tabular}{|c|c|c|c|c|c|}
\hline \multirow[t]{2}{*}{ Variáveis } & \multicolumn{2}{|c|}{ Caracol } & \multicolumn{2}{|c|}{ Anísio de Abreu } & \multirow{2}{*}{$p^{a}$} \\
\hline & $\mathrm{n}$ & $\%$ & $n$ & $\%$ & \\
\hline Escolaridade da mãe (anos) $(\mathrm{N}=1640)$ & & & & & 0,003 \\
\hline $0-4$ & 371 & 40,0 & 228 & 32,0 & \\
\hline $5-8$ & 336 & 36,2 & 278 & 39,1 & \\
\hline 9 ou mais & 221 & 23,8 & 206 & 28,9 & \\
\hline Escolaridade do pai (anos) $(\mathrm{N}=1373) \mathrm{b}$ & & & & & 0,17 \\
\hline $0-4$ & 425 & 54,5 & 293 & 49,4 & \\
\hline $5-8$ & 198 & 25,4 & 167 & 28,2 & \\
\hline 9 ou mais & 157 & 20,1 & 133 & 22,4 & \\
\hline Idade da mãe (anos) ( $N=1640)$ & & & & & 0,85 \\
\hline $13-19$ & 105 & 11,3 & 74 & 10,4 & \\
\hline $20-24$ & 349 & 37,6 & 281 & 39,5 & \\
\hline $25-29$ & 260 & 28,0 & 193 & 27,1 & \\
\hline 30 ou mais & 214 & 23,1 & 164 & 23,0 & \\
\hline No filhos $(N=1566)^{b}$ & & & & & 0,009 \\
\hline Nenhum & 359 & 40,8 & 303 & 44,3 & \\
\hline 1 & 279 & 31,6 & 227 & 33,2 & \\
\hline 2 & 129 & 14,6 & 101 & 14,7 & \\
\hline 3 ou mais & 115 & 13,0 & 53 & 7,8 & \\
\hline \multicolumn{6}{|l|}{ Socioeconômicas } \\
\hline Renda familiar $(S M) c \quad(N=1590)$ & & & & & 0,47 \\
\hline$<1$ & 633 & 72,1 & 466 & 65,4 & \\
\hline $1-1,9$ & 137 & 15,6 & 161 & 22,6 & \\
\hline $2-2,9$ & 68 & 7,7 & 59 & 8,3 & \\
\hline 3,0 ou mais & 40 & 4,6 & 26 & 3,7 & \\
\hline Abastecimento de água $(\mathrm{N}=1640)$ & & & & & $<0,001$ \\
\hline Não & 532 & 57,3 & 352 & 49,4 & \\
\hline Sim, dentro de casa & 183 & 19,7 & 288 & 40,5 & \\
\hline Sim, no terreno & 213 & 23,0 & 72 & 10,1 & \\
\hline Luz elétrica na casa $(\mathrm{N}=1640)$ & & & & & 0,005 \\
\hline Não & 100 & 10,8 & 48 & 6,7 & \\
\hline $\operatorname{sim}$ & 828 & 89,2 & 664 & 93,3 & \\
\hline Distância $(\mathrm{km})$ do serviço de saúde $(\mathrm{N}=1640)$ & & & & & 0,011 \\
\hline$>3$ & 422 & 45,5 & 279 & 39,2 & \\
\hline$\leq 3$ & 506 & 54,5 & 433 & 60,8 & \\
\hline
\end{tabular}


Distribuição da amostra de acordo com as variáveis para consulta em serviço de saúde nos últimos três meses de acordo com variáveis geográficas, demográficas, socioeconômicas, biológicas da criança, indicadoras de gravidade e de organização da família. Caracol e Anísio de Abreu, Piauí, Brasil, 2008 (N=1640).

\begin{tabular}{|c|c|c|c|c|c|}
\hline \multirow[t]{2}{*}{ Variáveis } & \multicolumn{2}{|c|}{ Caracol } & \multicolumn{2}{|c|}{ Anísio de Abreu } & \multirow{2}{*}{ pa } \\
\hline & $\mathrm{n}$ & $\%$ & $\mathrm{n}$ & $\%$ & \\
\hline \multicolumn{6}{|l|}{ Biológicas da criança } \\
\hline Sexo $(N=1640)$ & & & & & 0,46 \\
\hline Feminino & 468 & 50,4 & 346 & 48,6 & \\
\hline Masculino & 460 & 49,6 & 366 & 51,4 & \\
\hline Idade (meses) $(\mathrm{N}=1640)$ & & & & & 0,85 \\
\hline 0 a 11 & 182 & 19,6 & 149 & 20,9 & \\
\hline 12 a 23 & 181 & 19,5 & 132 & 18,5 & \\
\hline 24 a 35 & 200 & 21,6 & 157 & 22,1 & \\
\hline 36 a 47 & 165 & 17,8 & 133 & 18,7 & \\
\hline 48 a 59 & 200 & 21,5 & 141 & 19,8 & \\
\hline Cor da pele $(\mathrm{N}=1640)$ & & & & & $<0,001$ \\
\hline Branca & 141 & 15,2 & 211 & 29,6 & \\
\hline Parda & 740 & 79,7 & 459 & 64,5 & \\
\hline Preta & 47 & 5,1 & 42 & 5,9 & \\
\hline Estado nutricional $(\mathrm{N}=1612)^{b}$ & & & & & 0,97 \\
\hline Normal & 570 & 63,0 & 449 & 63,5 & \\
\hline Desnutrição & 159 & 17,6 & 123 & 17,4 & \\
\hline Sobrepeso/Obesidade & 176 & 19,5 & 135 & 19,1 & \\
\hline \multicolumn{6}{|l|}{ Organização da Família } \\
\hline Bolsa família ( $N=1640)$ & & & & & 0,24 \\
\hline Não & 544 & 58,6 & 397 & 55,8 & \\
\hline Sim & 384 & 41,4 & 315 & 44,2 & \\
\hline Cartão da criança $(\mathrm{N}=1640)$ & & & & & 0,09 \\
\hline Não & 58 & 6,3 & 31 & 4,4 & \\
\hline Sim & 870 & 93,8 & 681 & 95,7 & \\
\hline \multicolumn{6}{|l|}{ Indicadores de gravidade } \\
\hline Hospitalização no último ano $(\mathrm{N}=1640)$ & & & & & 0,10 \\
\hline Não & 825 & 88,9 & 614 & 86,2 & \\
\hline Sim & 103 & 11,1 & 98 & 13,8 & \\
\hline Morte de criança com $<5$ anos $(\mathrm{N}=1592)$ b & & & & & 0,52 \\
\hline $\operatorname{Sim}$ & 6 & 0,7 & 3 & 0,4 & \\
\hline Não & 888 & 99,3 & 695 & 99,6 & \\
\hline
\end{tabular}

a Valor de $p$ referente ao teste de qui-quadrado de Pearson; b Número total de observações diferente entre algumas variáveis devido a perdas de informação; c SM = salário mínimo mensal 
Tabela 2

Prevalência e razões de prevalências (RP) brutas para consulta em serviço de saúde nos últimos três meses de acordo com as variáveis geográficas, demográficas, socioeconômicas, biológicas da criança, indicadoras de gravidade e de organização da família. Caracol e Anísio de Abreu, Piauí, Brasil, 2008 (N=1640).

\begin{tabular}{|c|c|c|c|c|c|c|c|c|c|c|}
\hline \multirow{3}{*}{ Variáveis } & \multicolumn{4}{|c|}{ Caracol } & \multicolumn{6}{|c|}{ Anísio de Abreu } \\
\hline & & & RP & IC95\% & $p^{b}$ & $\begin{array}{r}\text { Pre } \\
\text { C }\end{array}$ & & RP & IC95\% & $p^{b}$ \\
\hline & $\mathrm{n}$ & $\%$ & & & & $\mathrm{n}$ & $\%$ & & & \\
\hline
\end{tabular}

Nível I

Geográficas

Área

Rural

248

248
162

$43,2 \quad 1,00$

$45,8 \quad 1,06 \quad 0,91-1,23$

0,44

0,09

157

188

$45,2 \quad 1,00$

$51,5 \quad 1,14 \quad 0,98-1,33$

162

Nível II

Características maternas/paternas

Cor da pele da mãe

Branca

Parda/mulata

Preta

Escolaridade da mãe (anos)

$0-4$

5-8

9 ou mais

Escolaridade do pai (anos) $(\mathrm{N}=1373)$

$0-4$

5-8

9 ou mais

Idade da mãe (anos)

13-19

20-24

25-29

30 ou mais

No filhos

Nenhum

1

2

3 ou mais

Socioeconômicas

Renda familiar (SM)b

$<1$

$1-1,9$

$2-2,9$

3,0 ou mais

0,47

Teste Wald para heterogeneidade de proporções ou tendência linear; b SM = salário mínimo mensal. 
Prevalência e razões de prevalências (RP) brutas para consulta em serviço de saúde nos últimos três meses de acordo com as variáveis geográficas, demográficas, socioeconômicas, biológicas da criança, indicadoras de gravidade e de organização da família. Caracol e Anísio de Abreu, Piauí, Brasil, 2008 (N=1640).

\begin{tabular}{|c|c|c|c|c|c|c|c|c|c|c|}
\hline \multirow{3}{*}{ Variáveis } & \multicolumn{4}{|c|}{ Caracol } & \multicolumn{6}{|c|}{ Anísio de Abreu } \\
\hline & \multicolumn{2}{|c|}{$\begin{array}{l}\text { Prevalência } \\
\text { Consulta }\end{array}$} & \multirow[t]{2}{*}{$\mathrm{RP}$} & \multirow[t]{2}{*}{ IC95\% } & \multirow[t]{2}{*}{$p^{b}$} & \multicolumn{2}{|c|}{$\begin{array}{c}\text { Prevalência } \\
\text { Consulta }\end{array}$} & \multirow[t]{2}{*}{$\mathrm{RP}$} & \multirow[t]{2}{*}{ IC95\% } & \multirow[t]{2}{*}{$p^{b}$} \\
\hline & $\mathrm{n}$ & $\%$ & & & & $\mathrm{n}$ & $\%$ & & & \\
\hline Abastecimento de água & & & & & 0,72 & & & & & 0,03 \\
\hline Não & 239 & 44,9 & 1,00 & - & & 161 & 45,7 & 1 & - & \\
\hline Sim, dentro de casa & 76 & 41,5 & 0,92 & $0,76-1,12$ & & 140 & 48,6 & 1,06 & $0,90-1,25$ & \\
\hline Sim, no terreno & 95 & 44,6 & 0,99 & $0,83-1,18$ & & 44 & 61,1 & 1,34 & $1,08-1,66$ & \\
\hline Luz elétrica na casa & & & & & 0,10 & & & & & 0,09 \\
\hline Não & 454 & 87,6 & 1,00 & - & & 336 & 91,6 & 1,00 & - & \\
\hline $\operatorname{sim}$ & 374 & 91,2 & 1,25 & $0,96-1,65$ & & 328 & 95,1 & 1,39 & $0,94-2,06$ & \\
\hline Distância $(\mathrm{km})$ do serviço de saúde & & & & & $<0,001$ & & & & & 0,52 \\
\hline$>3$ & 159 & 37,7 & 1,00 & - & & 131 & 47,0 & 1,00 & - & \\
\hline$\leq 3$ & 251 & 49,6 & 1,32 & $1,13-1,53$ & & 214 & 49,4 & 1,05 & $0,90-1,23$ & \\
\hline \multicolumn{11}{|l|}{ Biológicas da criança } \\
\hline Sexo & & & & & 0,37 & & & & & 0,51 \\
\hline Feminino & 200 & 42,7 & 1,00 & - & & 172 & 49,7 & 1,00 & - & \\
\hline Masculino & 210 & 45,7 & 1,07 & $0,92-1,23$ & & 173 & 47,3 & 0,95 & $0,88-1,11$ & \\
\hline Idade (meses) & & & & & 0,05 & & & & & $<0,001$ \\
\hline 0 a 11 & 88 & 48,4 & 1,00 & - & & 86 & 57,7 & 1,00 & - & \\
\hline 12 a 23 & 90 & 49,7 & 1,02 & $0,83-1,27$ & & 73 & 55,3 & 0,96 & $0,78-1,18$ & \\
\hline 24 a 35 & 85 & 42,5 & 0,88 & $0,71-1,10$ & & 72 & 45,9 & 0,79 & $0,64-0,99$ & \\
\hline 36 a 47 & 62 & 37,6 & 0,78 & $0,61-0,99$ & & 59 & 44,4 & 0,77 & $0,61-0,97$ & \\
\hline 48 a 59 & 85 & 42,5 & 0,88 & $0,71-1,10$ & & 55 & 39,0 & 0,68 & $0,53-0,87$ & \\
\hline Cor da pele & & & & & 0,15 & & & & & 0,68 \\
\hline Branca & 60 & 42,6 & 1,00 & - & & 100 & 47,4 & 1,00 & - & \\
\hline Parda & 336 & 45,4 & 1,07 & $0,87-1,31$ & & 227 & 49,5 & 1,04 & $0,88-1,24$ & \\
\hline Preta & 14 & 29,8 & 0,70 & $0,43-1,13$ & & 18 & 42,9 & 0,90 & $0,62-1,32$ & \\
\hline Estado nutricional & & & & & 0,52 & & & & & 0,57 \\
\hline Normal & 251 & 44,0 & 1,00 & - & & 214 & 47,7 & 1,00 & - & \\
\hline Desnutrição & 66 & 41,5 & 0,94 & $0,77-1,16$ & & 60 & 48,8 & 1,02 & $0,83-1,26$ & \\
\hline Sobrepeso/Obesidade & 84 & 47,7 & 1,08 & $0,91-1,30$ & & 68 & 50,4 & 1,05 & $0,87-1,28$ & \\
\hline \multicolumn{11}{|l|}{ Nível III } \\
\hline \multicolumn{11}{|l|}{ Organização da família } \\
\hline Bolsa Família & & & & & 0,20 & & & & & 0,92 \\
\hline Não & 231 & 42,5 & 1,00 & - & & 193 & 48,6 & 1,00 & - & \\
\hline $\operatorname{sim}$ & 179 & 46,6 & 1,10 & $0,95-1,27$ & & 152 & 48,3 & 0,99 & $0,85-1,16$ & \\
\hline Cartão da criança & & & & & 0,15 & & & & & 0,48 \\
\hline Não & 20 & 34,5 & 1,00 & - & & 13 & 41,9 & 1,00 & - & \\
\hline Sim & 390 & 44,8 & 1,30 & $0,90-1,87$ & & 332 & 48,8 & 1,16 & $0,76-1,77$ & \\
\hline
\end{tabular}

a Teste Wald para heterogeneidade de proporções ou tendência linear; b SM = salário mínimo mensal. 
Prevalência e razões de prevalências (RP) brutas para consulta em serviço de saúde nos últimos três meses de acordo com as variáveis geográficas, demográficas, socioeconômicas, biológicas da criança, indicadoras de gravidade e de organização da família. Caracol e Anísio de Abreu, Piauí, Brasil, 2008 ( $N=1640)$.

\begin{tabular}{|c|c|c|c|c|c|c|c|c|c|c|}
\hline \multirow{3}{*}{ Variáveis } & \multicolumn{4}{|c|}{ Caracol } & \multicolumn{6}{|c|}{ Anísio de Abreu } \\
\hline & \multicolumn{2}{|c|}{$\begin{array}{l}\text { Prevalência } \\
\text { Consulta }\end{array}$} & \multirow[t]{2}{*}{$\mathrm{RP}$} & \multirow[t]{2}{*}{ IC95\% } & \multirow[t]{2}{*}{$p^{b}$} & \multicolumn{2}{|c|}{$\begin{array}{l}\text { Prevalência } \\
\text { Consulta }\end{array}$} & \multirow[t]{2}{*}{ RP } & \multirow[t]{2}{*}{ IC95\% } & \multirow[t]{2}{*}{$p^{b}$} \\
\hline & $\mathrm{n}$ & $\%$ & & & & $\mathrm{n}$ & $\%$ & & & \\
\hline \multicolumn{11}{|l|}{ Nível IV } \\
\hline \multicolumn{11}{|l|}{ Indicadores de gravidade } \\
\hline Hospitalização no último ano & & & & & 0,09 & & & & & 0,20 \\
\hline Não & 357 & 43,3 & 1,00 & - & & 292 & 47,6 & 1,00 & - & \\
\hline $\operatorname{sim}$ & 53 & 51,5 & 1,19 & $0,97-1,46$ & & 53 & 54,1 & 1,14 & $0,93-1,39$ & \\
\hline Morte de criança com $<5$ anos & & & & & 0,75 & & & & & 0,64 \\
\hline $\operatorname{sim}$ & 3 & 50,0 & 1,00 & - & & 1 & 33,3 & 1,00 & - & \\
\hline Não & 391 & 44,0 & 0,88 & $0,39-1,97$ & & 338 & 48,6 & 1,46 & $0,29-7,25$ & \\
\hline
\end{tabular}

a Teste Wald para heterogeneidade de proporções ou tendência linear; b SM = salário mínimo mensal.

\section{Tabela 3}

Razões de prevalências (RP) ajustadas para consulta em serviço de saúde nos últimos três meses de acordo com variáveis geográficas, demográficas, socioeconômicas, biológicas da criança, indicadoras de gravidade e de organização da família. Caracol e Anísio de Abreu, Piauí, Brasil, 2008 ( $N=1640)$.

\begin{tabular}{|c|c|c|c|c|c|c|}
\hline \multirow{2}{*}{ Variáveis } & \multicolumn{3}{|c|}{ Caracol } & \multicolumn{3}{|c|}{ Anísio de Abreu } \\
\hline & RP & IC95\% & $p^{b}$ & RP & IC95\% & $p^{b}$ \\
\hline \multicolumn{7}{|l|}{ Nível la } \\
\hline \multicolumn{7}{|l|}{ Geográficas } \\
\hline Área & & & & & & 0,29 \\
\hline Rural & & & & 1,00 & - & \\
\hline Urbana & & & & 1,11 & $0,91-1,36$ & \\
\hline \multicolumn{7}{|l|}{ Nível Ila } \\
\hline \multicolumn{7}{|c|}{ Características maternas/paternas } \\
\hline Escolaridade da mãe (anos) & & & & & & 0,44 \\
\hline $0-4$ & & & & 1,00 & - & \\
\hline $5-8$ & & & & 1,21 & $0,98-1,49$ & \\
\hline 9 ou mais & & & & 1,10 & $0,87-1,39$ & \\
\hline Escolaridade do pai (anos) & & & 0,92 & & & \\
\hline $0-4$ & 1,00 & - & & & & \\
\hline $5-8$ & 1,02 & $0,84-1,25$ & & & c & \\
\hline 9 ou mais & 0,96 & $0,76-1,22$ & & & & \\
\hline Idade da mãe (anos) & & & 0,06 & & & 0,15 \\
\hline $13-19$ & 0,88 & $0,68-1,14$ & & 1,22 & $0,96-1,54$ & \\
\hline $20-24$ & 1,00 & - & & 1,00 & - & \\
\hline $25-29$ & 0,88 & $0,71-1,08$ & & 1,23 & $1,01-1,51$ & \\
\hline 30 ou mais & 0,78 & $0,60-1,02$ & & 1,15 & $0,90-1,47$ & \\
\hline
\end{tabular}


Razões de prevalências (RP) ajustadas para consulta em serviço de saúde nos últimos três meses de acordo com variáveis geográficas, demográficas, socioeconômicas, biológicas da criança, indicadoras de gravidade e de organização da família. Caracol e Anísio de Abreu, Piauí, Brasil, 2008 (N=1640).

\begin{tabular}{|c|c|c|c|c|c|c|}
\hline \multirow{2}{*}{ Variáveis } & \multicolumn{3}{|c|}{ Caracol } & \multicolumn{3}{|c|}{ Anísio de Abreu } \\
\hline & $\mathrm{RP}$ & IC95\% & $p^{b}$ & $\mathrm{RP}$ & IC95\% & $p^{b}$ \\
\hline No filhos & & & 0,63 & & & 0,03 \\
\hline Nenhum & 1,00 & - & & 1,00 & - & \\
\hline 1 & 0,94 & $0,77-1,13$ & & 0,86 & $0,71-1,05$ & \\
\hline 2 & 0,97 & $0,75-1,25$ & & 0,85 & $0,66-1,11$ & \\
\hline 3 ou mais & 0,90 & $0,65-1,25$ & & 0,80 & $0,56-1,14$ & \\
\hline \multicolumn{7}{|l|}{ Socioeconômicas } \\
\hline Renda familiar (SM)d & & & 0,03 & & c & \\
\hline$<1$ & 1,00 & - & & & & \\
\hline $1-1,9$ & 1,14 & $0,93-1,39$ & & & & \\
\hline $2-2,9$ & 1,10 & $0,78-1,54$ & & & & \\
\hline 3,0 ou mais & 1,53 & $1,12-2,09$ & & & & \\
\hline Abastecimento de água & c & & & & & 0,02 \\
\hline Não & & & & 1,00 & - & \\
\hline Sim, dentro de casa & & & & 0,98 & $0,79-1,21$ & \\
\hline Sim, no terreno & & & & 1,29 & $1,01-1,64$ & \\
\hline Luz elétrica na casa & & & 0,69 & & & 0,13 \\
\hline Não & 1,00 & - & & 1,00 & - & \\
\hline $\operatorname{sim}$ & 0,96 & $0,72-1,27$ & & 1,31 & $0,87-1,96$ & \\
\hline Distância $(\mathrm{km})$ do serviço de saúde & & & $<0,001$ & & c & \\
\hline$>3$ & 1 & - & & & & \\
\hline$\leq 3$ & 1,37 & $1,15-1,64$ & & & & \\
\hline \multicolumn{7}{|l|}{ Biológicas da criança } \\
\hline Idade (meses) & & & 0,17 & & & 0,001 \\
\hline 0 a 11 & 1,00 & - & & 1,00 & - & \\
\hline 12 a 23 & 1,07 & $0,85-1,34$ & & 1,00 & $0,82-1,23$ & \\
\hline 24 a 35 & 0,88 & $0,70-1,12$ & & 0,84 & $0,67-1,06$ & \\
\hline 36 a 47 & 0,82 & $0,62-1,08$ & & 0,81 & $0,63-1,04$ & \\
\hline 48 a 59 & 0,92 & $0,71-1,19$ & & 0,72 & $0,55-0,94$ & \\
\hline Cor da pele & & & 0,32 & c & & \\
\hline Branca & 1,00 & - & & & & \\
\hline Parda & 1,17 & $0,92-1,47$ & & & & \\
\hline Preta & 0,96 & $0,59-1,56$ & & & & \\
\hline \multicolumn{7}{|l|}{ Nível IIla } \\
\hline \multicolumn{7}{|l|}{ Organização da família } \\
\hline Cartão da criança & & & 0,14 & c & & \\
\hline Não & 1,00 & - & & & & \\
\hline Sim & 1,30 & $0,91-1,85$ & & & & \\
\hline \multicolumn{7}{|l|}{ Nível IVa } \\
\hline \multicolumn{7}{|l|}{ Indicadores de gravidade } \\
\hline Hospitalização no último ano & & & 0,06 & c & & \\
\hline Não & 1,00 & - & & & & \\
\hline Sim & 1,21 & $0,99-1,48$ & & & & \\
\hline
\end{tabular}

a Variáveis de cada nível ajustadas entre si e ajustadas e para as variáveis com $p<0,05$ do nível superior conforme modelo de determinação;

b Teste Wald para heterogeneidade de proporções ou tendência linear;

c Variáveis não inseridas no modelo de análise multivariada da respectiva cidade devido valor de $p \geq 0,20$ na análise bruta;

d SM = salário mínimo mensal. 
vista. Por sua vez, as crianças a partir de 48 meses de idade consultaram menos (Tabela 3 ).

\section{Discussão}

Uma das principais preocupações na saúde mundial incentivada pela Organização Mundial da Saúde e brasileira inclusive como dispositivo constitucional tem sido cobertura universal de assistência. ${ }^{18}$ Existem evidências mostrando que a ampliação da cobertura na assistência leva a melhores resultados na saúde da população pela ampliação da oferta de serviços públicos, extensão de programas sociais e disseminação de intervenções específicas.19,20 Entretanto, no presente estudo, praticamente metade das crianças havia consultado nos três meses anteriores à entrevista, o que permitiu fazer a estimativa de duas consultas/criança ano. Ao levar em consideração que foram incluídas crianças menores de um ano, as quais exigiam maior acompanhamento e, ressaltando que as crianças acima de um ano de idade também necessitavam de maiores cuidados de saúde, inferiu-se que a concentração de consultas foi muito baixa. De fato, a análise mostrou que as crianças menores de 11 meses de idade consultaram mais os serviços de saúde como aliás, era esperado uma vez que o instrumento de pesquisa não discriminava consultas preventivas (pesar, realizar imunizações) daquelas por motivos de doenças.

Outro objetivo dos sistemas de saúde tem sido a distribuição equitativa dos recursos. ${ }^{21}$ Neste estudo, foram encontrados indícios de iniquidade. Em Caracol, as crianças cujas famílias possuíam maior renda, consultavam mais. Em Anísio Abreu, as diferenças por renda e escolaridade não foram verificadas, o que poderia indicar uma desejável ausência de desigualdade na distribuição dos recursos. Devese destacar que a análise da variável escolaridade paterna ficou prejudicada pelo elevado percentual de respostas ignoradas. Entretanto, em Anísio de Abreu nas famílias abastecidas por água (um bem essencial para a vida), as crianças consultaram mais. Esta variável pode ser considerada marcadora das condições socioeconômicas e, então, revelar iniquidade no município. ${ }^{22}$ Assim, mesmo no presente estudo realizado em dois lugares muito pobres, com sociedades muito homogêneas, a análise possibilitou encontrar diferenças nos cuidados à saúde relacionados às condições socioeconômicas. ${ }^{23-25}$ Aliás, estudo transversal realizado em Caracol em 2005 tinha mostrado as pessoas inseridas no mais baixo tercil de pobreza apresentando piores indicadores de cobertura. 26

No município de Caracol, as crianças que residiam em regiões próximas aos serviços de saúde consultaram mais nos três meses anteriores à entrevista. Este resultado sugeriu maior disponibilidade na oferta dos serviços para estas crianças. À medida que aumentava a distância geográfica entre os usuários e o serviço diminuía o número de consultas. 27,28

A análise mostrou que as crianças que foram hospitalizadas no último ano também apresentaram maiores prevalência na utilização dos serviços de saúde, com significância limítrofe. Hospitalizações estão associadas à gravidade das condições clínicas e a severidade da situação pode impor maior acompanhamento e aumento do número de consultas. ${ }^{29}$

Os resultados deste estudo apontaram que, em Caracol, mães com idade igual ou superior a 30 anos buscaram menos o serviço de saúde, embora com significância limítrofe. Contudo, em Anísio de Abreu os achados foram divergentes: mães de 25 a 29 anos consultaram mais, com seus filhos, conforme os limites dos intervalos de confiança. Deve-se chamar a atenção para que esta associação entre idade da mãe e consulta em serviço de saúde foi verificada mesmo ajustando-se para número de filhos. Sabe-se que mulheres com mais de 30 anos tendem a apresentar uma maior prevalência de pré-natal inadequado. Isto sugere que essas mães têm menor cuidado com sua saúde. Assim, deve ser ressaltado que do ponto de vista de provimento de cuidados em saúde, a busca ativa das mães com mais de 30 anos pode ser estratégica para a qualificação dos cuidados de saúde infantil.

A idade das crianças estava associada à prevalência de consultas. Nos dois municípios, principalmente em Anísio de Abreu, foi observada a diminuição de consultas a partir dos 24 meses, o que seria esperado pela diminuição da morbidade e pelo desenvolvimento somático da população infantil.

O estado nutricional de todos os participantes do estudo foi classificado mediante o índice de massa corpórea, o que o Programa Anthro permite. Embora as crianças menores de dois anos tradicionalmente sejam classificadas pelo índice peso para altura, a análise não mostrou associação. 30

Deve-se ressaltar a presença de algumas limitações relacionadas à natureza deste estudo, como causalidade reversa, viés de memória e problemas de representação, próprias de estudos transversais. No entanto, foi possível identificar a magnitude das variáveis avaliando a realização de consultas nos serviços de saúde e definindo estratégias de ação. Obteve-se rigor na seleção do método e na condução do trabalho de campo. Esses critérios garantiram a validade interna e podem ser representativos para 
municípios deste porte e características do Brasil.

Estudos transversais podem fornecer importantes contribuições para o diagnóstico de condições de saúde. O presente estudo realizado em dois municípios muito pobres no país mostrou dificuldades no acesso aos serviços de saúde, como indicado pela

\section{Referências}

1. Victora CG, Cesar JA. Saúde materno-infantil no Brasil: padrões de morbidade e possíveis intervenções. In: Rouquayrol MZ, Almeida-Filho NM. Epidemiologia e saúde. 6 ed. Rio de Janeiro (RJ): MEDSI; 2003.

2. Kerr-Pontes LR, Rouquayrol MZ. Medida da saúde coletiva. In: Rouquayrol MZ, Almeida-Filho NM. Epidemiologia e saúde. 6 ed. Rio de Janeiro (RJ): MEDSI; 2003

3. Ramos DD, Lima MADS. Acesso e acolhimento aos usuários em uma unidade de saúde de Porto Alegre, Rio Grande do Sul, Brasil. Cad Saúde Pública. 2003; 19 (1): 27 34.

4. Marmot M. Health in an unequal world. Lancet. 2006; 368 2081-94.

5. Starfield B, Shi L, Macinko J. Contribution of primary care to health systems and health. Milbank Q 2005; 83: 457-502.

6. Barros FC, Matijasevich A, Requejo JH, Giugliani E, Maranhão AG, Monteiro CA, Barros AJD, Bustreo F, Merialdi M, Victora CG. Recent trends in maternal, newborn and child health in Brazil: progress toward Millenium Development Goals 4 and 5. Am J Public Health. 2010; 100 (10): 1877-89

7. Victora CG, Aquino EML, Leal MC, Monteiro CA, Barros FC, Szwarcwald CL. Saúde de mães e crianças no Brasil progressos e desafios. Lancet. (Série Brasil) [Internet] 2011; 32-46. Disponível em: http://download thelancet.com/flatcontentassets/pdfs/brazil/brazilpor2.pdf

8. César JA, Matijasevich A, Santos IS, Barros AJD, Dias da Costa JS, Barros FC, Victora CG. The use of maternal and child health services in three population-based cohorts in Southern Brazil, 1982-2004. Cad Saúde Pública. 2008; 24 (Supl. 3): 427-36.

9. Schramm JMA, Szwarcwald CL. Diferenciais nas taxas de mortalidade neonatal e natimortalidade hospitalares no Brasil: um estudo com base no Sistema de Informações Hospitalares do Sistema Único de Saúde (SIH/SUS). Cad Saúde Pública. 2000; 16 (4): 1031-40.

10. Cesar JA, Gonçalves TS, Neumann N, Oliveira- Filho JA, Diziekaniak AC. Saúde infantil em áreas pobres das Regiões Norte e Nordeste do Brasil: comparando indicadores básicos em áreas atendidas pela Pastoral da Criança e áreas-controle. Cad Saúde Pública. 2005; 21: 1845-55.

11. IBGE (Instituto Brasileiro de Geografia e Estatística). IBGE Cidades - PI, 2010. Rio de Janeiro: IBGE. Disponível em http://www.ibge.gov.br/cidadesat/ topwindow.htm?1.

12. PNUD (Programa das Nações Unidas para o Desenvolvimento). Atlas do Desenvolvimento Humano. Índice de Desenvolvimento Humano - Municipal, 1991 e 2000 [serial online] [cited $2011 \mathrm{dez}$ 7]. Disponível em baixa concentração de consultas. $\mathrm{O}$ estudo revelou fatores associados que expressavam iniquidade à saúde. Os serviços de atenção primária deveriam reconhecer a população infantil como um de seus grupos de acompanhamento prioritário.

http://www.pnud.org.br/atlas/ranking/IDH-M $\% 2091 \% 2000$ $\% 20$ Ranking\%20decrescente $\% 20$ (pelos $\% 20$ dados $\%$ 20de\%202000).htm

13. DATASUS (Departamento de Informática do Sistema Único de Saúde). Informações de Saúde. Óbitos Infantis Piauí [serial online] 2010 [cited 2014 mar 10]. Available from http://tabnet.datasus.gov.br/cgi/tabcgi.exe?sim/ cnv/obt10PI.def

14. WHO (World Health Organization). WHO Anthro for personal computers: software for assessing growth and development of the world's children (version 3.2.2). Geneva, Switzerland: 2009. [acesso em 29 mar 2012]. Available from: http://www.who.int/childgrowth/ software/en/

15. WHO Multicentre Growth Reference Study Group. WHO child growth standards based on length/height, weight and age. Acta Paediatr. 2006; 450 (Suppl.): 76-85.

16. Victora CG, Huttly SR, Fuchs SC, Olinto MTA. The role of conceptual frameworks in epidemiological analysis: a hierarchical approach. Int J Epidemiol 1997; 26 (1): 224-47.

17. Barros AJD, Hirakata UM. Alternatives for logistic regression in cross-sectional studies: an empirical comparision of models that directly estimate the prevalence ratio. BMC Med Res Methodol. 2003; 3: 21

18. Moreno-Serra R, Smith PC. Does progress towards universal health coverage improve population health? Lancet. 2012; 380: 917-23.

19. Giedion U, Diaz BY. A review of evidence. In: Escobar ML, Griffin C, Shaw RP (editors). The impact of health insurance in low and middle income countries. Washington D.C.: Brookings Institution Press; 2010. p. 13-32.

20. Nyman J. Health insurance theory: the case of the missing welfare gain. Eur J Health Econ. 2008; 9 (4): 369-80.

21. WHO Task Force on Research Priorities for Equity in Health, WHO Equity Team. Priorities for research to take forward the health equity policy agenda. Bull World Health Organ. 2005; 83 (12): 948-53.

22. Paim J, Travassos C, Almeida C, Bahia L, Macinko J. O sistema de saúde brasileiro: história, avanços e desafios. Lancet. (Série Brasil) [Internet]. 2011; 11-31. Disponível em: http://download.thelancet.com/flatcontentassets/ pdfs/brazil/brazilpor1.pdf

23. Castro MSM, Travassos C, Carvalho MS. Fatores associados às internações hospitalares no Brasil. Ciênc Saúde Coletiva. 2002; 7: 687-708.

24. Travassos C, Viacava F, Fernandes C, Almeida CM Desigualdades geográficas e sociais na utilização de serviços de saúde no Brasil. Ciênc Saúde Coletiva. 2000; 5: 133-49. 
25. Almeida C, Travassos C, Porto S, Labra ME. Health sector reform in Brazil: a case study of inequity. Int J Health Serv. 2000; 30: 129-62.

26. Mano PS, Cesar JA, González-Chica DA, Neumann NA. Iniquidade na assistência à gestação e ao parto em município do semiárido brasileiro. Rev Bras Saúde Matern Infant. 2011; 11 (4): 381-8.

27. Mooney C, Zwanziger J, Phibbs CS, Schmitt S. Is travel distance a barrier to veteran's use of VA hospitals for medical surgical care? Soc Sci Med. 2000; 50: 1743-55.

28. Oliveira EXG, Travassos C, Carvalho MS. Acesso à inter-

Recebido em 11 de julho 2013

Versão final apresentada em 20 de outubro de 2014

Aprovado em 3 de novembro de 2014 nação hospitalar nos municípios brasileiros em 2000: territórios do Sistema Único de Saúde. Cad Saúde Pública. 2004; 20 (Suppl. 2): 298-309.

29. Dias da Costa JS, Olinto MTA, Gigante DP, Menezes AMB, Macedo S, Daltoé T, Santos IS, Fuchs SC. Utilização de serviços ambulatoriais de saúde em Pelotas, Rio Grande do Sul, Brasil: alguns fatores relacionados com as consultas médicas acima da média. Cad Saúde Pública. 2008; 24 (2): 353-63.

30. Meller FO, Araújo CLP, Madruga SW. Fatores associados ao excesso de peso em crianças brasileiras menores de cinco anos. Ciênc Saúde Coletiva. 2014; 19 (3): 943-55. 